\title{
Nuclear shape transitions and elastic magnetic electron scattering
}

\author{
B. Hernández $\odot,{ }^{1,2}$ P. Sarriguren $\odot,,^{1, *}$ O. Moreno, ${ }^{2}$ E. Moya de Guerra $\odot,{ }^{2}$ D. N. Kadrev $\odot,{ }^{3}$ and A. N. Antonov $\circledast^{3}$ \\ ${ }^{1}$ Instituto de Estructura de la Materia, IEM-CSIC, Serrano 123, E-28006 Madrid, Spain \\ ${ }^{2}$ Departamento de Estructura de la Materia, Física Térmica y Electrónica, and IPARCOS, Facultad de Ciencias Físicas, \\ Universidad Complutense de Madrid, Madrid E-28040, Spain \\ ${ }^{3}$ Institute for Nuclear Research and Nuclear Energy, Bulgarian Academy of Sciences, Sofia 1784, Bulgaria
}

(Received 27 October 2020; accepted 7 December 2020; published 6 January 2021)

\begin{abstract}
Backward elastic electron scattering from odd- $A$ nuclear targets is characterized by magnetic form factors containing precise information on the nuclear structure. We study the sensitivity of the magnetic form factors to structural effects related to the evolution and shape transitions in both isotopic and isotonic chains. Calculations of magnetic form factors are performed in the plane-wave Born approximation. The nuclear structure is obtained from a deformed self-consistent mean-field calculation based on a Skyrme HF + BCS formalism. Collective effects are included in the cranking approximation, whereas nucleon-nucleon correlations are taken into account in the coherent density fluctuation model. The evolution of the magnetic form factors is found to exhibit signatures of shape transitions that show up in selected isotopic and isotonic chains involving both stable and unstable nuclei. Several cases are identified as suitable candidates for showing such fingerprints of shape transitions. A new generation of electron scattering experiments involving electron-radioactive beam colliders will be available in the near future, leading to a renewed interest in this field.
\end{abstract}

DOI: 10.1103/PhysRevC.103.014303

\section{INTRODUCTION}

Electron scattering from nuclei has been well established in the past as a tool to provide detailed information on nuclear charge and current distributions [1-4]. It is well known that the weakness of the electromagnetic interaction relative to the nuclear force, as well as its accurate quantum electrodynamics description, result in electron scattering being a suitable and powerful tool to study nuclear charge distributions and radii, transition probabilities, momentum distributions, and spectroscopic factors from elastic, inelastic, and quasielastic channels [5-7].

Electron scattering can be also used as an additional tool to complement the information obtained from other probes. This could be the case of magnetic dipole $(M 1)$ excitations in nuclei, where it is well known that complementary information is obtained when using different electromagnetic $\left(\gamma, \gamma^{\prime}\right),\left(e, e^{\prime}\right)$ or hadronic $\left(p, p^{\prime}\right)$ probes $[8,9]$. Electron scattering may also be used as a tool to investigate weak processes, such as parity-violating electron scattering [10-12] or other processes involving neutrinos [13,14].

Although charge scattering is in most cases the dominant contribution to the cross section, electrons also interact with the nuclear electromagnetic current distributions, whose contributions can be isolated with proper choices of the kinematical conditions of the process. The experimental observation of the electric and magnetic form factors provides information on the convection and magnetization currents

\footnotetext{
*p.sarriguren@csic.es
}

within the nucleus. In particular, elastic magnetic electron scattering provides fine details of the nuclear ground-state current and magnetization distributions [15-17].

Contrary to the case of charge scattering, where all the protons contribute coherently to the nuclear response, the magnetic scattering response in odd- $A$ nuclei depends to a large extent on the single-particle properties of the valence nucleon wave function, whereas the collective aspects give minor contributions in most cases, but may play an important role in modulating the single-particle contributions. In addition, magnetic electron scattering may provide information on both protons and neutrons on an equal footing, since their intrinsic magnetic moments are similar in magnitude. Similarly to the case of charge scattering, magnetic scattering has been studied in stable nuclei from different theoretical frameworks [15], including shell model [18-21], relativistic mean field [22], and deformed mean-field models [16,23-31].

In a previous work [29], we showed that the plane-wave Born approximation (PWBA) together with a self-consistent deformed Skyrme HF + BCS formalism for the nuclear structure calculation are able to reproduce the experimental form factors of both spherical and deformed stable nuclei. Therefore, we have at hand a reliable procedure to address the study of unstable nuclei and to predict the magnetic form factors of nuclei not yet measured. Based on this method, we plan in this work to study the sensitivity of the magnetic form factors against structural changes of the nucleus. The best scenario for this study involves chains of odd- $A$ isotopes with odd $Z$ or chains of odd- $A$ isotones with odd $N$, whose ground-state spin/parity $\left(I^{\pi}\right)$, determined by the unpaired nucleon, only changes if the structure of the nucleus is changed. 
Therefore, we look first for experimental fingerprints of structural changes and identify some candidates where this study could be of particular interest. The structural change will be related to a shape transition along the chain [32,33].

The study of isotopic or isotonic chains involves in general unstable nuclei. One criterion for our selection of the chains to be studied is based on the eventual feasibility of electron scattering experiments from the nuclei in the chain. Therefore, we focus on the unstable nuclei that are close to stability, in addition to the stable ones. The experimental difficulties found when dealing with unstable targets have limited the present knowledge to stable nuclei, but it is expected that the new generation of electron-ion colliders at radioactive nuclear beam facilities [34] will overcome some of the technical difficulties. This is the case of ELISe (FAIR-GSI) [35] and SCRIT (RIKEN) [36]. The conceptual design and the scientific challenges of the electron-ion collider ELISe (Electron-Ion Scattering in a Storage Ring) can be found in Ref. [37]. In SCRIT (Self-Confining Radioactive Ion Target) a circulating beam of electrons scatters off ions stored in a trap [38,39]. In this facility, an elastic electron scattering experiment on ${ }^{132} \mathrm{Xe}$ has already been performed [40], demonstrating its feasibility. Electron scattering on unstable nuclei could be a tool to study the evolution of the charge distributions in isotopic chains and therefore, to test theoretical models aiming to predict nuclear charge distributions. Examples of these studies can be found in Refs. [41-46].

The structure of this article is the following. In Sec. II we present the theoretical framework and basic expressions to calculate the magnetic form factors in deformed nuclei. We also introduce the spherical limit of these calculations, as well as the effects of short-range correlations in this limit. Section III contains the results obtained. The latter are given, first for static moments and then for the magnetic form factors in the isotopic and isotonic chains selected. Section IV contains the conclusions of the work.

\section{THEORETICAL FORMALISM}

\section{A. Electron scattering form factors}

The basic aspects of the formalism of electron scattering from deformed nuclei that we follow in this work was introduced earlier [16,24]. The work in Ref. [24] demonstrated for the first time that deformation has to be included in the theoretical description to reproduce the experimental data for the deformed nucleus ${ }^{181} \mathrm{Ta}$. Since then, the method has been applied to different cases [25-29], where the sensitivities of the results to different approximations concerning nuclear structure and reaction mechanism were studied. In particular, it has been shown that the magnetic form factors of deformed nuclei may differ considerably from those of spherical nuclei [29]. Here, we briefly summarize this formalism. Following the notation of Ref. [16], the general cross section for ultrarelativistic electron scattering for transitions from the nuclear ground state $\left(I_{i}\right)$ to final states $\left(I_{f}\right)$ in PWBA and for unpolarized projectiles and unoriented targets, is given by

$$
\left.\frac{d \sigma}{d \Omega}\right|_{I_{i} \rightarrow I_{f}}=4 \pi \sigma_{M} f_{\mathrm{rec}}^{-1}\left[V_{L}\left|F_{L}\right|^{2}+V_{T}\left|F_{T}\right|^{2}\right],
$$

in terms of the Mott cross section

$$
\sigma_{M}=\left[\frac{\alpha \cos (\theta / 2)}{2 \epsilon_{i} \sin ^{2}(\theta / 2)}\right]^{2},
$$

and a recoil factor $f_{\text {rec }}$. The cross section is separated into longitudinal (L) and transverse (T) parts, weighted with different kinematical factors. The dependence on the electron kinematics is given by the $\mathrm{L}$ and $\mathrm{T}$ Rosenbluth factors,

$$
V_{L}=\left(Q^{2} / q^{2}\right)^{2}, \quad V_{T}=\tan ^{2}(\theta / 2)-\left(Q^{2} / 2 q^{2}\right),
$$

where the kinematical variables are defined so that an incident electron with four-momentum $k_{i \mu}=\left(\epsilon_{i}, \boldsymbol{k}_{i}\right)$ is scattered from the nucleus through an angle $\theta$ to four-momentum $k_{f \mu}=$ $\left(\epsilon_{f}, \boldsymbol{k}_{f}\right)$ by exchanging a virtual photon with four-momentum $Q=(\omega, \boldsymbol{q})$.

Whereas the longitudinal form factors receive coherent contributions from all the charged nucleons, the transverse form factors are basically single-particle observables that depend mostly on the properties of the unpaired nucleon in the outermost shell. Hence, the longitudinal contribution (charge scattering) is dominant in most cases and the transverse contribution can only be disentangled using special kinematical conditions. In particular, backward scattering $\left(\theta=180^{\circ}\right)$ is commonly used to measure $F_{T}$.

The dependence on the nuclear structure is contained in the $q$-dependent longitudinal and transverse form factors, which are written in terms of Coulomb $(\mathrm{C})$, transverse electric $(\mathrm{E})$, and transverse magnetic (M) multipoles,

$$
\left|F_{L}\right|^{2}=\sum_{\lambda \geqslant 0}\left|F^{C \lambda}\right|^{2}, \quad\left|F_{T}\right|^{2}=\sum_{\lambda \geqslant 1}\left[\left|F^{M \lambda}\right|^{2}+\left|F^{E \lambda}\right|^{2}\right],
$$

which are defined as the reduced matrix elements of the multipole operators $\hat{T}^{\sigma \lambda}$ between initial and final nuclear states

$$
\left|F^{\sigma \lambda}\right|^{2}=\frac{\left|\left\langle I_{f}|| \hat{T}^{\sigma \lambda}(q)|| I_{i}\right\rangle\right|^{2}}{2 I_{i}+1} .
$$

For elastic scattering, parity and time reversal invariance imply that only even Coulomb and odd transverse magnetic multipoles contribute. Then, at $\theta=180^{\circ}$ only odd magnetic multipoles survive in PWBA,

$$
\left|F_{T}(q)\right|^{2}=\sum_{\lambda=\text { odd }}\left|F^{M \lambda}\right|^{2} .
$$

The magnetic multipole operators are defined as

$$
\hat{T}_{\mu}^{M \lambda}(q)=i^{\lambda} \int d \mathbf{r} j_{\lambda}(q r) \mathbf{Y}_{\lambda \lambda}^{\mu}\left(\Omega_{r}\right) \cdot \hat{\mathbf{J}}(\mathbf{r}),
$$

where $\hat{\mathbf{J}}(\mathbf{r})$ is the current density operator that contains both convection and magnetization components arising from the motion and from the intrinsic magnetic moments of the nucleons, respectively.

Center of mass and finite nucleon size corrections are included in the calculations. For the center of mass correction we use the usual factor obtained in the harmonic-oscillator approximation, $f_{\text {c.m. }}=\exp \left(q^{2} A^{-2 / 3} / 4\right)$. In the magnetization currents, we use bare nucleon magnetic moments, $\mu_{s}^{p}=2.793 \mu_{N}, \mu_{s}^{n}=-1.913 \mu_{N}$, corrected with dipole form 
factors [47]. In the convection currents, we use bare orbital $g$-factors, $g_{\ell}^{p}=1$ and $g_{\ell}^{n}=0$, scaled by $q$-dependent form factors given by a sum of monopoles parametrized in Ref. [47] for the proton and by the difference of two Gaussians [48] for the neutron.

The effects of Coulomb distortion could be evaluated quantitatively in the distorted-wave Born approximation (DWBA) with a phase-shift calculation [49]. Nevertheless, neglecting the Coulomb distortion offers clear advantages for the analysis and interpretation of experimental data on magnetic scattering and PWBA is commonly used. In PWBA the connection between data and the underlying physical quantities is more transparent and calculations are simplified. The most important effect of Coulomb distortion is accounted for by using an effective momentum transfer. The general procedure is to convert the experimental form factors into plane-wave form factors that can be directly compared with PWBA calculations, as was done in Ref. [15].

\section{B. Mean field and form factors in a deformed formalism}

The ground state of an axially symmetric deformed nucleus is characterized by its angular momentum $I$, its projection along the symmetry axis $k$, and parity $\pi$. Initial and final states are the same for elastic scattering $\left(I k^{\pi}\right)$.

The magnetic multipole form factors $F^{M \lambda}$ can be written in terms of intrinsic form factors $\mathcal{F}^{M \lambda}$ weighted by angular momentum dependent coefficients. To lowest order in an expansion in powers of the total angular momentum, the intrinsic multipoles depend only on the intrinsic structure of the ground-state band [16]. The transition multipoles in Eq. (6) for the elastic case $I_{f}=I_{i}=k$ can be written as

$$
\begin{aligned}
\left.F^{M \lambda}\right|_{\text {def }}= & \langle k k \lambda 0 \mid k k\rangle \mathcal{F}_{k}^{M \lambda}+\langle k-k \lambda 2 k \mid k k\rangle \mathcal{F}_{2 k}^{M \lambda} \\
& +\sqrt{\frac{\lambda(\lambda+1)}{2}}\langle k k \lambda 0 \mid k k\rangle \mathcal{F}_{R}^{M \lambda} .
\end{aligned}
$$

As we can see from the expression above, the magnetic form factors in odd $A$ nuclei receive two types of contributions, single-particle $\left(\mathcal{F}_{k}^{M \lambda}, \mathcal{F}_{2 k}^{M \lambda}\right)$ and collective $\left(\mathcal{F}_{R}^{M \lambda}\right)$. The singleparticle multipoles depend only on the single-particle intrinsic wave function of the odd nucleon if the even-even core is time-reversal invariant, as we assume in this work. They are different from zero only for $k \neq 0$ bands and are given by

$$
\begin{gathered}
\mathcal{F}_{k}^{M \lambda}=\left\langle\phi_{k}\left|\hat{T}_{0}^{M \lambda}\right| \phi_{k}\right\rangle, \\
\mathcal{F}_{2 k}^{M \lambda}=\left\langle\phi_{k}\left|\hat{T}_{2 k}^{M \lambda}\right| \phi_{\bar{k}}\right\rangle+\delta_{k, 1 / 2} \frac{a}{\sqrt{2}} \mathcal{F}_{R}^{M \lambda} .
\end{gathered}
$$

$\hat{T}_{\mu}^{M \lambda}$ is the $\mu$ component of the $M \lambda$ tensor operator (see Eq. (7) and Ref. [2]). $\phi_{k}$ and $\phi_{\bar{k}}$ are the intrinsic wave functions of the odd nucleon and its time reversed, respectively, and $a=$ $\left\langle\phi_{k}\left|j_{+}\right| \phi_{\bar{k}}\right\rangle$ is the decoupling parameter for $k=1 / 2$ bands.

$\mathcal{F}_{R}^{M \lambda}$ are the magnetic multipoles of the collective rotational current (rotational multipoles) that depend on the nuclear rotational model used to describe the band [16]. These contributions were studied [26,27] by using different microscopic and macroscopic models, concluding that they are, in general, small compared to single-particle contributions. They are only expected to be relevant in the $M 1$ multipoles at low $q$ and do not differ much from one rotational model to another. Thus, we opted here for the cranking model, that produces better moments of inertia.

The nuclear structure is described within a self-consistent axially symmetric deformed $\mathrm{HF}+\mathrm{BCS}$ formalism. The wave function for the $i$ single-particle state is written in terms of the spin components $\phi_{i}^{+}$and $\phi_{i}^{-}$as [50]

$$
\begin{aligned}
\phi_{i}(\mathbf{R}, \sigma)= & \phi_{i}^{+}(r, z) \exp \left(\mathrm{i} \Lambda^{-} \varphi\right) \chi_{+}(\sigma) \\
& +\phi_{i}^{-}(r, z) \exp \left(\mathrm{i} \Lambda^{+} \varphi\right) \chi_{-}(\sigma) .
\end{aligned}
$$

The variables $r, z$, and $\varphi$ are the cylindrical coordinates of the radius-vector $\mathbf{R}$. $\chi_{ \pm}(\sigma)$ are the spin wave functions and $\Lambda^{ \pm}=\Omega_{i} \pm 1 / 2 \geqslant 0$, where $\Omega_{i}$ is the projection along the symmetry axis of the total angular momentum, and it characterizes the single-particle Hartree-Fock solutions for axially symmetric deformed nuclei, together with parity $\pi_{i}$.

The wave functions $\phi_{i}$ are expanded into eigenfunctions $\psi_{\alpha}(\mathbf{R}, \sigma)$ of an axially deformed harmonic oscillator potential using eleven major shells,

$$
\phi_{i}(\mathbf{R}, \sigma)=\sum_{\alpha} C_{\alpha}^{i} \psi_{\alpha}(\mathbf{R}, \sigma),
$$

with $\alpha=\left\{n_{r}, n_{z}, \Lambda, \Sigma\right\}$. All the results presented in this work correspond to the Skyrme interaction SLy4 [51], which has been thoroughly tested on many nuclear properties along the full nuclear chart.

In the present work, the mean field of the odd- $A$ nucleus is generated within the equal filling approximation (EFA), a prescription used in self-consistent mean-field calculations for odd- $A$ nuclei that preserves time-reversal invariance. In this approximation half of the unpaired nucleon sits in a given orbital and the other half in the time-reversed partner. The odd nucleon orbital, characterized by $\Omega_{i}=k$ and $\pi_{i}$, is chosen among those around the Fermi level, according to the experimental ground-state spin and parity values. It is worth mentioning that with this choice of spin and parity we obtain the minimum of the energy in most cases. In the few cases where the minimum corresponds to a different spin and parity, these assignments are found experimentally very close to the ground states.

The explicit expressions for all the intrinsic form factors in Eqs. (9) and (10) in terms of these wave functions can be found in Refs. [16,24]. Expressions for the intrinsic rotational multipoles $\mathcal{F}_{R}^{M \lambda}$ can be also found in Ref. [16] for different microscopic and macroscopic models.

Nuclei with quadrupole deformation parameters $\left|\beta_{p}\right| \leqslant$ 0.06 are considered in this work as spherical and the spherical limit of the present deformed formalism is applied to them. In Tables I and II these cases are identified with the label "sph" in the column for quadrupole deformations. Thus, for these nuclei we first constrain the deformed calculation to zero deformation. In the spherical limit there are no collective magnetic multipoles $\left(\mathcal{F}_{R}^{M \lambda}=0\right)$ and the form factors are related to the deformed ones by

$$
\left.F^{M \lambda}\right|_{\text {sph limit }}=\left.\left[\eta_{j}^{\lambda}\right]^{-1} F^{M \lambda}\right|_{\text {def }},
$$


TABLE I. Experimental spin/parity $\left(I^{\pi}\right)$ and half-life $\left(T_{1 / 2}\right)$, calculated and measured charge root-mean-square radii $\left(r_{c, \text { th }}\right.$ and $r_{c, \text { exp }}$ [57] in $\mathrm{fm})$, calculated quadrupole deformation $\left(\beta_{p}\right)$, calculated and measured spectroscopic electric quadrupole moment $\left(Q_{\text {lab,th }}\right.$ and $Q_{\text {lab,exp }}[58]$ in barns), and calculated (in spherical limit and deformed) and measured magnetic moments ( $\mu_{\mathrm{th}, \mathrm{sph}}, \mu_{\mathrm{th}, \mathrm{def}}, \mu_{\exp }[58]$ in $\left.\left[\mu_{N}\right]\right)$ in isotopic chains with $Z=9,13,23,25,53$, and 55 .

\begin{tabular}{|c|c|c|c|c|c|c|c|c|c|c|}
\hline Nucleus & $I^{\pi}$ & $T_{1 / 2}$ & $r_{c, \mathrm{th}}$ & $r_{c, \exp }$ & $\beta_{p}$ & $Q_{\text {lab,th }}$ & $Q_{\text {lab,exp }}$ & $\mu_{\mathrm{th}, \mathrm{sph}}$ & $\mu_{\mathrm{th}, \mathrm{def}}$ & $\mu_{\exp }$ \\
\hline${ }^{19} \mathrm{~F}$ & $1 / 2^{+}$ & stable & 2.8591 & $2.8976(25)$ & +0.193 & 0 & - & +4.793 & +2.275 & $+2.628868(8)$ \\
\hline${ }^{25} \mathrm{Al}$ & $5 / 2^{+}$ & $7.183 \mathrm{~s}$ & 3.1317 & - & +0.303 & +0.166 & - & +4.793 & +3.795 & $3.6455(12)$ \\
\hline${ }^{27} \mathrm{Al}$ & $5 / 2^{+}$ & stable & 3.0948 & $3.0610(31)$ & +0.200 & +0.107 & $+0.1466(10)$ & +4.793 & +3.768 & $+3.6415069(7)$ \\
\hline${ }^{29} \mathrm{Al}$ & $5 / 2^{+}$ & $6.56 \mathrm{~min}$ & 3.0934 & - & +0.128 & +0.069 & - & +4.793 & +3.743 & - \\
\hline${ }^{53} \mathrm{~V}$ & $7 / 2^{-}$ & $1.543 \mathrm{~min}$ & 3.6388 & - & $\mathrm{sph}$ & sph & - & +5.793 & - & - \\
\hline${ }^{51} \mathrm{Mn}$ & $5 / 2^{-}$ & $46.2 \mathrm{~min}$ & 3.7235 & $3.7026(212)$ & +0.244 & +0.367 & - & +5.793 & +3.591 & $3.5683(13)$ \\
\hline${ }^{53} \mathrm{Mn}$ & $7 / 2^{-}$ & $3.7 \times 10^{6} \mathrm{yr}$ & 3.6897 & $3.6662(76)$ & $\mathrm{sph}$ & $\mathrm{sph}$ & - & +5.793 & - & $5.024(7)$ \\
\hline${ }^{55} \mathrm{Mn}$ & $5 / 2^{-}$ & stable & 3.7316 & $3.7057(22)$ & +0.212 & +0.321 & $+0.33(1)$ & +5.793 & +3.532 & $+3.46871790(9)$ \\
\hline${ }^{125} \mathrm{I}$ & $5 / 2^{+}$ & $59.4 \mathrm{~d}$ & 4.7507 & - & -0.148 & -0.779 & $-0.776(17)$ & +1.717 & +3.219 & $2.821(5)$ \\
\hline${ }^{135} \mathrm{Cs}$ & $7 / 2^{+}$ & $2.3 \times 10^{6} \mathrm{yr}$ & 4.8107 & $4.8067(47)$ & $\mathrm{sph}$ & $\mathrm{sph}$ & $+0.050(2)$ & +1.717 & - & $+2.7324(2)$ \\
\hline
\end{tabular}

with $\left.F^{M \lambda}\right|_{\text {def }}$ as in Eq. (8) and $\eta_{j}^{\lambda}$ given by [29]

$$
\eta_{j}^{\lambda}=\langle j j \lambda 0 \mid j j\rangle^{2}\left[1+\delta_{\lambda, 2 j} \frac{\langle j-j \lambda 2 j \mid j j\rangle^{2}}{\langle j j \lambda 0 \mid j j\rangle^{2}}\right] .
$$

It was found in Ref. [29] that the results from a standard spherical formalism agree perfectly with the results obtained in the spherical limit of the present deformed formalism.

\section{Correlation effects in the spherical limit}

Many experimental nuclear data show sizable effects of nucleon-nucleon $(\mathrm{NN})$ correlations that cannot be accounted for within mean-field approximations [52,53]. However, the single-particle picture can be recovered with methods involving NN correlations by using the natural orbital representation [54] of the one-body density matrix (OBDM). The natural orbitals and occupation numbers are obtained by diagonalizing the OBDM solving the equation

$$
\int d \mathbf{r}^{\prime} \rho\left(\mathbf{r}, \mathbf{r}^{\prime}\right) \psi_{\alpha}\left(\mathbf{r}^{\prime}\right)=n_{\alpha} \psi_{\alpha}(\mathbf{r}),
$$

where $\psi_{\alpha}(\mathbf{r})$ are the natural orbitals and $n_{\alpha}$ are the natural occupation numbers.

In the present work, we include correlations within the Coherent Density Fluctuation Model (CDFM) $[52,53,55,56]$. The OBDM in this model has the form

$$
\rho\left(\mathbf{r}, \mathbf{r}^{\prime}\right)=\int|\mathcal{F}(x)|^{2} \rho_{x}\left(\mathbf{r}, \mathbf{r}^{\prime}\right) d x
$$

where

$$
\rho_{x}\left(\mathbf{r}, \mathbf{r}^{\prime}\right)=3 \rho_{0}(x) \frac{j_{1}\left(k_{F}(x)\left|\mathbf{r}-\mathbf{r}^{\prime}\right|\right)}{k_{F}(x)\left|\mathbf{r}-\mathbf{r}^{\prime}\right|} \Theta\left(x-\frac{\left|\mathbf{r}+\mathbf{r}^{\prime}\right|}{2}\right),
$$

TABLE II. Same as in Table I, but for isotonic chains with $N=9,11,25$, and 57 .

\begin{tabular}{lcccccccccc}
\hline \hline Nucleus & $I^{\pi}$ & $T_{1 / 2}$ & $r_{c \text {,th }}$ & $r_{c, \text { exp }}[57]$ & $\beta_{p}$ & $Q_{\text {lab,th }}$ & $Q_{\text {lab,exp }}[58]$ & $\mu_{\text {th,sph }}$ & $\mu_{\text {th,def }}$ & $\mu_{\text {exp }}[58]$ \\
\hline${ }^{15} \mathrm{C}$ & $1 / 2^{+}$ & $2.449 \mathrm{~s}$ & 2.5764 & - & +0.122 & 0 & - & -1.913 & -1.361 & $1.720(9)$ \\
${ }^{17} \mathrm{O}$ & $5 / 2^{+}$ & stable & 2.7476 & $2.6932(75)$ & sph & sph & -0.02578 & -1.913 & - & $-1.89379(9)$ \\
${ }^{19} \mathrm{Ne}$ & $1 / 2^{+}$ & $17.22 \mathrm{~s}$ & 2.9543 & $3.0082(40)$ & +0.189 & 0 & - & -1.913 & -1.521 & $-1.88542(8)$ \\
${ }^{19} \mathrm{O}$ & $5 / 2^{+}$ & $26.88 \mathrm{~s}$ & 2.7445 & - & $\mathrm{sph}$ & $\mathrm{sph}$ & $0.0037(4)$ & -1.913 & - & $1.53195(7)$ \\
${ }^{21} \mathrm{Ne}$ & $3 / 2^{+}$ & stable & 2.9582 & $2.9695(33)$ & +0.276 & +0.058 & $+0.103(8)$ & -1.913 & -0.718 & $-0.661797(5)$ \\
${ }^{23} \mathrm{Mg}$ & $3 / 2^{+}$ & $11.317 \mathrm{~s}$ & 3.1136 & - & +0.398 & +0.112 & $0.125(5)$ & -1.913 & -0.732 & $0.5364(3)$ \\
${ }^{45} \mathrm{Ca}$ & $7 / 2^{-}$ & $162.61 \mathrm{~d}$ & 3.4973 & $3.4944(21)$ & $\mathrm{sph}$ & $\mathrm{sph}$ & $+0.046(14)$ & -1.913 & - & $-1.3274(14)$ \\
${ }^{47} \mathrm{Ti}$ & $5 / 2^{-}$ & stable & 3.6072 & $3.5962(19)$ & +0.185 & +0.230 & $+0.30(2)$ & -1.913 & -0.816 & $-0.78848(1)$ \\
${ }^{49} \mathrm{Cr}$ & $5 / 2^{-}$ & $42.3 \mathrm{~min}$ & 3.6932 & - & +0.260 & +0.369 & - & -1.913 & -0.841 & $0.476(3)$ \\
${ }^{99} \mathrm{Mo}$ & $1 / 2^{+}$ & $65.976 \mathrm{~h}$ & 4.3769 & - & $\mathrm{sph}$ & 0 & - & +1.488 & - & $0.375(3)$ \\
${ }^{101} \mathrm{Ru}$ & $5 / 2^{+}$ & stable & 4.4486 & $4.4606(20)$ & +0.148 & +0.565 & $+0.46(2)$ & +1.488 & -0.921 & $-0.719(6)$ \\
${ }^{103} \mathrm{Pd}$ & $5 / 2^{+}$ & $16.991 \mathrm{~d}$ & 4.4936 & - & +0.161 & +0.656 & - & +1.488 & -0.799 & - \\
\hline \hline
\end{tabular}


with

$$
\rho_{0}(x)=\frac{3 A}{4 \pi x^{3}}, \quad k_{F}(x)=\left(\frac{3 \pi^{2}}{2} \rho_{0}(x)\right)^{1 / 3} .
$$

The weight function $|\mathcal{F}(x)|^{2}$ in Eq. (16) in the case of monotonically decreasing density distributions can be obtained from the density as

$$
|\mathcal{F}(x)|^{2}=-\left.\frac{1}{\rho_{0}(x)} \frac{d \rho(r)}{d r}\right|_{r=x} .
$$

The effect of these correlations has been studied in previous works [29,53], showing that they shift the tails of the form factors to higher momentum transfer, improving the agreement with experiment. We have included these correlations in the calculations on the magnetic form factors in spherical nuclei. Although not shown here, we have also calculated the correlations on the spherical limit of the deformed nuclei, finding a similar effect in the tails of the magnetic form factors. As we shall see, this effect does not change the clear differences observed in the profiles of the form factors from different $I^{\pi}$ and deformations that manifest themselves mainly at low momentum transfer.

\section{RESULTS AND DISCUSSION}

In this section we study several isotopic and isotonic chains that have been chosen according to the criteria discussed above. We first consider vanadium and aluminum isotopes as examples of the general expected behavior within isotopic chains with a fixed number of odd protons and a variable number of even neutrons. In principle, one expects to have the same spin and parity for all the members of the chain, as well as a similar deformation, which in our case is spherical for vanadium and prolate for aluminum isotopes. As we shall see, the profiles of the calculated magnetic form factors are quite similar as well. However, the isotopic chains of fluorine, manganese, iodine, and cesium under study here are characterized by a change in the angular momentum of the ground states related to a shape transition. The corresponding magnetic form factors also exhibit a great sensitivity to these structural changes. The isotonic chains with $N=9,11,25$, and 57 are also chosen as examples with angular momentum changes and the magnetic form factors show also very different profiles.

\section{A. Static moments}

Tables I and II show theoretical and experimental groundstate properties of the considered isotopic and isotonic chains, respectively. Namely, spins and parities, half-lives, charge root-mean-square radii $r_{c}$, quadrupole proton deformations $\beta_{p}$, spectroscopic electric quadrupole moments $Q_{\mathrm{lab}}$, and magnetic moments $\mu$. The results are compared with experimental data from Ref. [57] for radii and from Ref. [58] for quadrupole and magnetic moments. Note that the lack of a sign in the experimental $Q$ and $\mu$ means that it is still undetermined.
The relationship between the intrinsic quadrupole moment $Q_{0}$ and the quadrupole deformation parameter $\beta_{p}$ is given by

$$
Q_{0}=\sqrt{\frac{5}{\pi}} Z e\left(r_{p}^{2}\right\rangle \beta_{p}
$$

where $\left\langle r_{p}^{2}\right\rangle$ is the proton mean-square radius. The measured quadrupole moment $Q_{\text {lab }}$ is related to the intrinsic quadrupole moment $Q_{0}$ by

$$
Q_{\mathrm{lab}}=\frac{3 k^{2}-I(I+1)}{(I+1)(2 I+3)} e Q_{0} .
$$

The measured electric quadrupole moments in the tables correspond to ground states $I=k$. Note that in the cases $I=k=$ $1 / 2, Q_{\text {lab }}=0$.

The magnetic moments in the deformed case $\mu_{\text {th,def }}$ are obtained from the expression,

$$
\begin{aligned}
\mu_{I}= & g_{R} I+\frac{k^{2}}{I+1}\left[g_{k}-g_{R}\right. \\
& \left.+\delta_{k, 1 / 2}(2 I+1)(-1)^{I+1 / 2} \sqrt{2} g_{2 k}\right],
\end{aligned}
$$

where $g_{R}$ is approximated by $Z / A$ and $g_{k}, g_{2 k}$ are defined in Ref. [16]. We also show for comparison the Schmidt values $\mu_{\text {th,sph }}$ obtained in the spherical limit.

Similarly to the cases studied in Ref. [29], we find here that the discrepancies between the calculated and the measured $r_{c}$ are just of a few per thousand in most cases. Only in the lightest nuclei the errors are somewhat larger, but always below two percent. In the case of the isotopic chains we can see that the charge radii increase slightly as the number of neutrons increase. This is a general trend expected from the tendency of protons to overlap maximally with neutrons and thus spreading the spatial distribution of the latter (see, e.g., Ref. [42]). This general trend is nevertheless altered by local effects related to deformation. A clear example of this peculiar behavior can be seen in the manganese isotopes, where the shape changes from prolate in ${ }^{51} \mathrm{Mn}$ to spherical in the magic $(N=28){ }^{53} \mathrm{Mn}$, and again to prolate in ${ }^{55} \mathrm{Mn}$. This structural change makes the charge radii decrease from ${ }^{51} \mathrm{Mn}$ to ${ }^{53} \mathrm{Mn}$ and increase from ${ }^{53} \mathrm{Mn}$ to ${ }^{55} \mathrm{Mn}$. In the case of the isotonic chains, as compared with the isotopic chains, we observe a more dramatic increase of the charge radii as the number of protons increases. This is expected as the protons occupy outer orbitals.

The quadrupole moments are calculated for the deformed nuclei and compared with the measured $Q_{\text {lab. They agree in }}$ sign and magnitude.

Concerning magnetic moments, we show the Schmidt values as well as the values from Eq. (22) in the deformed cases. We see that the Schmidt values reproduce reasonably well the magnetic moments of spherical nuclei, whereas for the deformed nuclei there is a clear improvement of the agreement with experiment using the deformed formalism.

\section{B. Form factors in isotopic chains}

In the case of even- $Z$ and odd- $N$ isotopes the spin and parity of the ground state, which depend on the odd-neutron state, change from isotope to isotope. Therefore, we expect 
rather different form factors as we move in an isotopic chain of this kind. On the contrary, in the case of even- $N$ and odd- $Z$ isotopes that we study in this work, the spin/parity of the ground state remain unchanged as long as there are no critical changes in the nuclear structure. The magnetic form factors of these isotopes are expected to be similar because they are mainly determined by the wave function of the odd proton, that does not change much. Therefore, changes in the spin/parity of the isotopes in these chains become signatures of structural transitions induced by the collective effect of the different number of neutrons on the odd-proton wave function. As it will be shown here, these effects can also be studied through the magnetic form factors.

The spherical limit defined earlier is applied to those nuclei with very small $\beta_{p}$ values. This limit involves a spherical constraint of the mean-field calculation, a redefinition of the multipole form factors given by Eq. (13), and the removal of collective contributions $\mathcal{F}_{R}^{M \lambda}$ from rotations of the core.

In the following figures for the isotopic chains under study in this work, we show in the top panels (a) a comparison of the total magnetic form factors corresponding to the three nuclei considered in each chain. In the lower panels (b), (c), and (d) we show, for each member of the chain, the total magnetic form factor decomposed into the contributing multipolarities. In the case of spherical nuclei we include the results of the CDFM calculations in the total magnetic form factor.

\section{Vanadium isotopes}

In Fig. 1 we show the magnetic form factors in isotopes of vanadium $(Z=23),{ }^{49,51,53} \mathrm{~V}$. They are examples of three spherical isotopes with the same observed ground-state spins and parities (see Table I). They are $I^{\pi}=7 / 2^{-}$odd- $A$ and odd- $Z$ nuclei, where the valence proton sits in the $f_{7 / 2}$ shell. Because of the similar nuclear structure in the three isotopes, the magnetic form factors in Fig. 1 are quite similar and therefore this chain is an example of the expected behavior of odd- $Z$ even- $N$ isotopes. Figures 1(b), 1(c) and 1(d) show the multipole decomposition of the total magnetic form factors, where the lowest multipole $(M 1)$ determines the low- $q$ behavior of the magnetic form factors, whereas the highest multipole, $M 7$ in this case, determines the large- $q$ tails. The other multipoles contribute in different degrees to the intermediate region of momentum transfer. This is generally true for all the cases under study. The experimental data on ${ }^{51} \mathrm{~V}$ from Refs. $[15,59,60]$ are well reproduced by the calculations.

Nucleon-nucleon correlations included in the CDFM approach are shown in the three spherical isotopes. They influence the form factor beyond $q=2 \mathrm{fm}^{-1}$ and the main effect is to increase the form factor at high momentum transfer. The agreement with experiment in the case of ${ }^{51} \mathrm{~V}$ is clearly improved by these correlations.

\section{Aluminum isotopes}

In Fig. 2 we show the magnetic form factors in isotopes of aluminum $(Z=13),{ }^{25,27,29} \mathrm{Al}$. The three isotopes are found experimentally to have $I^{\pi}=5 / 2^{+}$in their ground states with the odd proton occupying the $d_{5 / 2}$ shell in the spherical limit. In the deformed model the odd proton occupies a
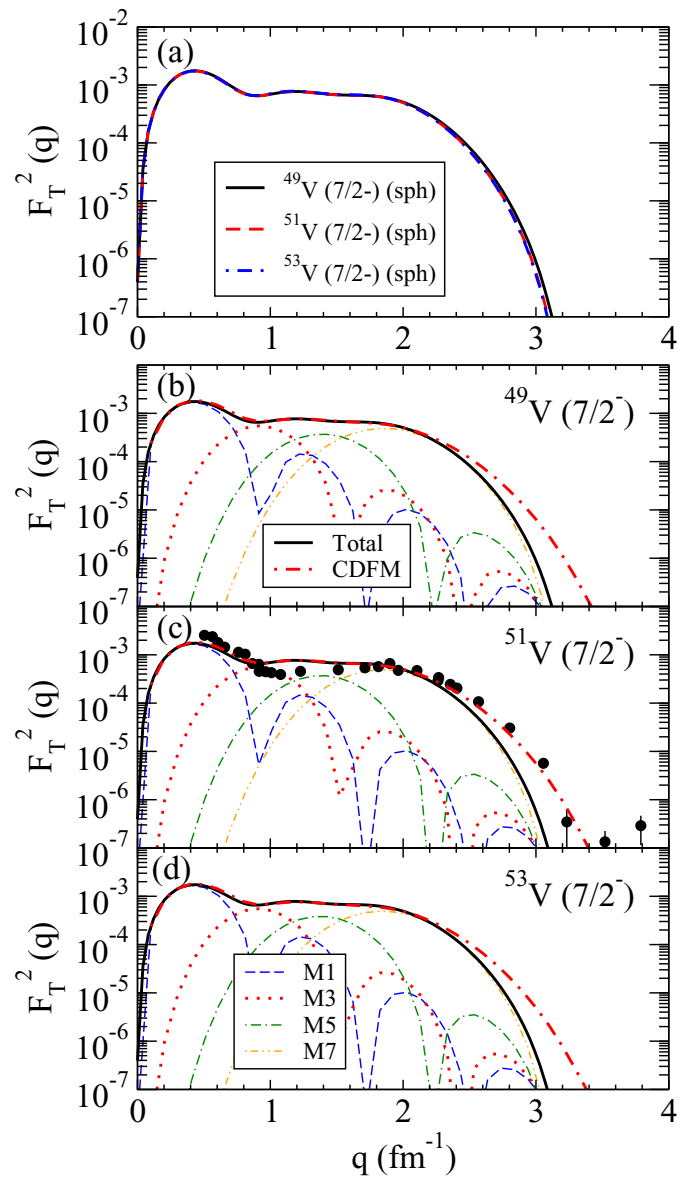

FIG. 1. Total magnetic form factors for the $Z=23$ isotopes ${ }^{49,51,53} \mathrm{~V}$ (a) and their decomposition into the contributing multipoles for ${ }^{49} \mathrm{~V}$ (b), ${ }^{51} \mathrm{~V}$ (c), and ${ }^{53} \mathrm{~V}$ (d). CDFM calculations are shown for the spherical cases. Data are taken from Refs. [15,59,60].

$k^{\pi}=5 / 2^{+}$prolate state or a $k^{\pi}=1 / 2^{+}$oblate state. Because the former is energetically favored in the three isotopes, our description corresponds to a prolate shape with the odd proton in a Nilsson-like state with asymptotic quantum numbers [202]5/2. The three isotopes have a similar deformation (see Table I) and the magnetic form factors are practically indistinguishable. Thus, adding couples of neutrons in this isotopic chain does not change the structure of the odd-proton state. This chain is another example of the expected behavior of odd- $Z$ even- $N$ isotopes, but in this case for deformed nuclei. The multipole decomposition shows that the multipole $M 1$ determines the behavior below $q=1 \mathrm{fm}^{-1}$ and the multipole $M 5$ determines the behavior beyond, whereas the intermediate multipole $M 3$ is irrelevant.

Experimental data for ${ }^{27} \mathrm{Al}$ from Refs. [15,61,62] are well reproduced, except in the high- $q$ region where the data are underestimated due to the lack of short-range correlations.

\section{Fluorine isotopes}

The case of fluorine isotopes $(Z=9),{ }^{17,19,21} \mathrm{~F}$, is different and much more interesting from a nuclear structure point of view. Experimentally, the ground states of ${ }^{17} \mathrm{~F}$ and ${ }^{21} \mathrm{~F}$ have 

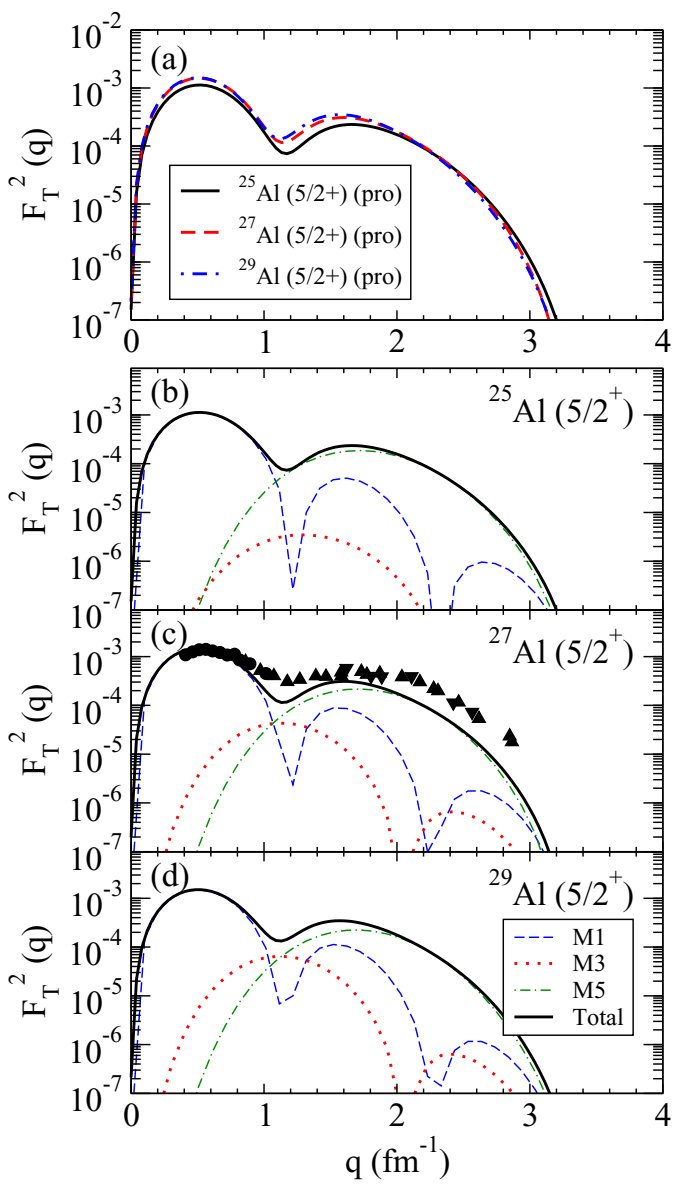

FIG. 2. Same as described in the caption of Fig. 1, but for the $Z=13$ isotopes ${ }^{25,27,29} \mathrm{Al}$. Data are taken from Refs. [15,61,62].

$I^{\pi}=5 / 2^{+}$, but ${ }^{19} \mathrm{~F}$ has $I^{\pi}=1 / 2^{+}$. In a simple Nilsson diagram one can see that the odd proton sits on the $d_{5 / 2}$ spherical shell either on the orbital $k^{\pi}=5 / 2^{+}$in the oblate region or on the orbital $k^{\pi}=1 / 2^{+}$in the prolate region. Since a prolate configuration is favored energetically in ${ }^{19} \mathrm{~F}$, the odd proton is expected to occupy the Nilsson orbital [220]1/2. However, the oblate shape is preferred in ${ }^{21} \mathrm{~F}$, and the odd proton occupies in this case the Nilsson orbital [202]5/2.

In the top panel of Fig. 3 we can see the total magnetic form factors of the three isotopes together. A clear difference can be observed among them related to the effect of deformation between the spherical ${ }^{17} \mathrm{~F}$ and the deformed ${ }^{19,21} \mathrm{~F}$, but also related to the different angular momentum of the ground states that gives rise to different multipole contributions. In panels (b), (c), and (d) we can see separately the multipole contributions in the cases of ${ }^{17} \mathrm{~F},{ }^{19} \mathrm{~F}$, and ${ }^{21} \mathrm{~F}$, respectively. The data in ${ }^{19} \mathrm{~F}$ are from Refs. [63,64]. The only multipole is $M 1$ and exhibits a three-peaked structure in this region of momentum transfer, which seems to be also the case with the data.

The different $I^{\pi}$ of the ground states observed as we move in the isotopic chain indicates a structural change. In the deformed picture this effect is associated with a shape transition from spherical $\left(5 / 2^{+}\right)$in ${ }^{17} \mathrm{~F}$ to prolate $\left(1 / 2^{+}\right)$in ${ }^{19} \mathrm{~F}$ and to oblate $\left(5 / 2^{+}\right)$in ${ }^{21} \mathrm{~F}$. Thus, the neutron environment of
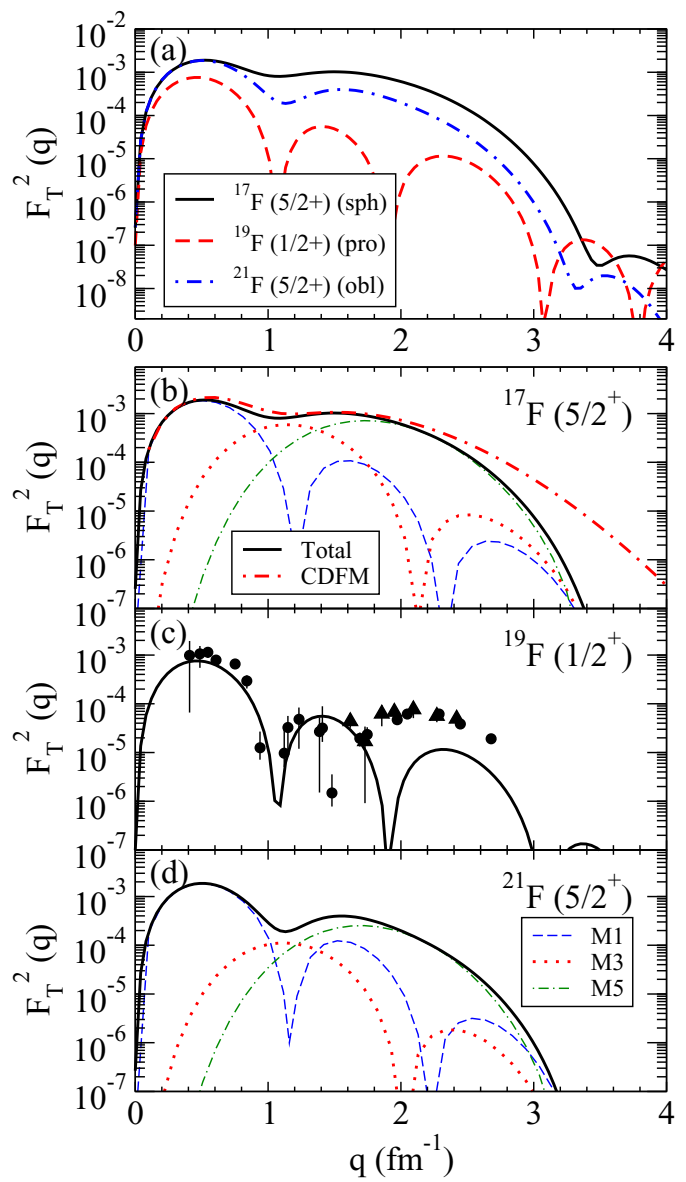

FIG. 3. Same as described in the caption of Fig. 1, but for the $Z=9$ isotopes ${ }^{17,19,21} \mathrm{~F}$. Data are taken from Refs. [63,64].

the three isotopes, $N=8,10,12$, determines the state where the odd proton sits. The self-consistent deformations in these isotopes can be seen in Table I. This structural change is translated into the magnetic form factor, which is indeed sensitive to the odd-proton state. The result is that in ${ }^{17} \mathrm{~F}$ and ${ }^{21} \mathrm{~F}$, the multipoles $M 3$ and especially $M 5$ contribute by filling the high- $q$ region and a two-peaked structure is obtained, rather different to the profile of the ${ }^{19} \mathrm{~F}$ isotope that exhibits a threepeaked structure.

The configurations with $1 / 2^{+}$and $5 / 2^{+}$in the three isotopes are indeed quite close in energy, competing to each other for being ground states. It is worth noting that experimentally, a $5 / 2^{+}$excited state is observed in ${ }^{19} \mathrm{~F}$ at an energy $E=197 \mathrm{keV}$. Similarly, $1 / 2^{+}$excited states are observed in both ${ }^{17} \mathrm{~F}$ and ${ }^{21} \mathrm{~F}$ at energies $E=495 \mathrm{keV}$ and $E=280$ $\mathrm{keV}$, respectively. These features point at a shape coexistence between spherical, oblate, and prolate configurations or to a mixture of them. Given the sensitivity of the magnetic form factors to the details of the odd-proton wave function, elastic scattering experiments could be used to gain information on these properties.

Pure single-particle results produce already a good agreement with experiment in the first peak of ${ }^{19} \mathrm{~F}$. Core contributions may not be very reliable in such a light nucleus, but we have included them in the rigid rotor model 
that improves slightly the agreement with experiment. Our calculations reproduce the structure of three peaks of the form factor measured although the third peak is underestimated. However, the nucleon-nucleon correlations calculated in the spherical nucleus ${ }^{17} \mathrm{~F}$ that increase the form factors beyond $q=2 \mathrm{fm}^{-1}$ suggest that a similar effect would be expected in the deformed nucleus ${ }^{19} \mathrm{~F}$ improving the agreement with experiment in the region of the third peak.

\section{Manganese isotopes}

The case of manganese isotopes $(Z=25),{ }^{51,53,55} \mathrm{Mn}$, is also an interesting example of a shape transition, which is already anticipated by the change in the angular momentum of their ground states, namely, $5 / 2^{-}$in ${ }^{51} \mathrm{Mn}, 7 / 2^{-}$in ${ }^{53} \mathrm{Mn}$, and $5 / 2^{-}$in ${ }^{55} \mathrm{Mn}$. This change is related to a shape transition from prolate in ${ }^{51} \mathrm{Mn}$ to spherical in ${ }^{53} \mathrm{Mn}$ (associated to the magic number $N=28$ ) and again to prolate in ${ }^{55} \mathrm{Mn}$, as can be seen in Table I. The odd proton occupies the spherical shell $f_{7 / 2}$, while the prolate configurations in ${ }^{51} \mathrm{Mn}$ and ${ }^{55} \mathrm{Mn}$ correspond to a Nilsson-like state with asymptotic quantum numbers [312]5/2.

Low-lying $7 / 2^{-}$excited states are observed experimentally in ${ }^{51} \mathrm{Mn}$ and ${ }^{55} \mathrm{Mn}$ at $E=237 \mathrm{keV}$ and $E=126 \mathrm{keV}$, respectively. A $5 / 2^{-}$excited states is also found in ${ }^{53} \mathrm{Mn}$ at $E=378 \mathrm{keV}$, showing again a competition between different configurations.

In Fig. 4(a) we can see the total magnetic form factors of the three isotopes, where a very clear difference between the spherical $\left(7 / 2^{-}\right){ }^{53} \mathrm{Mn}$ isotope and the prolate deformed $\left(5 / 2^{-}\right){ }^{51,55} \mathrm{Mn}$ isotopes is observed. Whereas the deformed isotopes ${ }^{51,55} \mathrm{Mn}$ show a first peak at $q=0.4 \mathrm{fm}^{-1}$ and a second peak, one order of magnitude smaller, centered at $q=1.2 \mathrm{fm}^{-1}$. The spherical isotope ${ }^{53} \mathrm{Mn}$ shows a small peak at $q=0.4 \mathrm{fm}^{-1}$ followed by a plateau-like structure up to about $q=2 \mathrm{fm}^{-1}$. Therefore, this is a very clear example of a structural change to which the magnetic scattering will be extremely sensitive.

The CDFM calculation on the spherical isotope ${ }^{53} \mathrm{Mn}$ shows again an increase of the tails of the form factors at high momentum transfer.

\section{Iodine isotopes}

The magnetic form factors of iodine isotopes $(Z=53)$ ${ }^{125,127,129} \mathrm{I}$ are plotted in Fig. 5. The measured angular momenta are $5 / 2^{+}$in ${ }^{125} \mathrm{I}, 5 / 2^{+}$in ${ }^{127} \mathrm{I}$, and $7 / 2^{+}$in ${ }^{129} \mathrm{I}$. This change is related to a shape transition from oblate in ${ }^{125,127} \mathrm{I}$ to spherical in ${ }^{129} \mathrm{I}$, as can be seen in Table I. Excited states $7 / 2^{+}$ in ${ }^{125} \mathrm{I}, 7 / 2^{+}$in ${ }^{127} \mathrm{I}$, and $5 / 2^{+}$in ${ }^{129} \mathrm{I}$ are also found at energies $E=114 \mathrm{keV}, E=58 \mathrm{keV}$, and $E=28 \mathrm{keV}$, respectively. The odd proton occupies the spherical shell $g_{7 / 2}$ in ${ }^{129} \mathrm{I}$, while it occupies the Nilsson oblate state [413]5/2 in ${ }^{125,127}$ I.

The magnetic form factor in ${ }^{129} \mathrm{I}$ shows a smoother profile than the form factors of ${ }^{125,127} \mathrm{I}$, which is caused by the enhancement of the intermediate multipoles in the spherical case. CDFM results are shown in ${ }^{129} \mathrm{I}$, but they are very similar to the mean-field results.
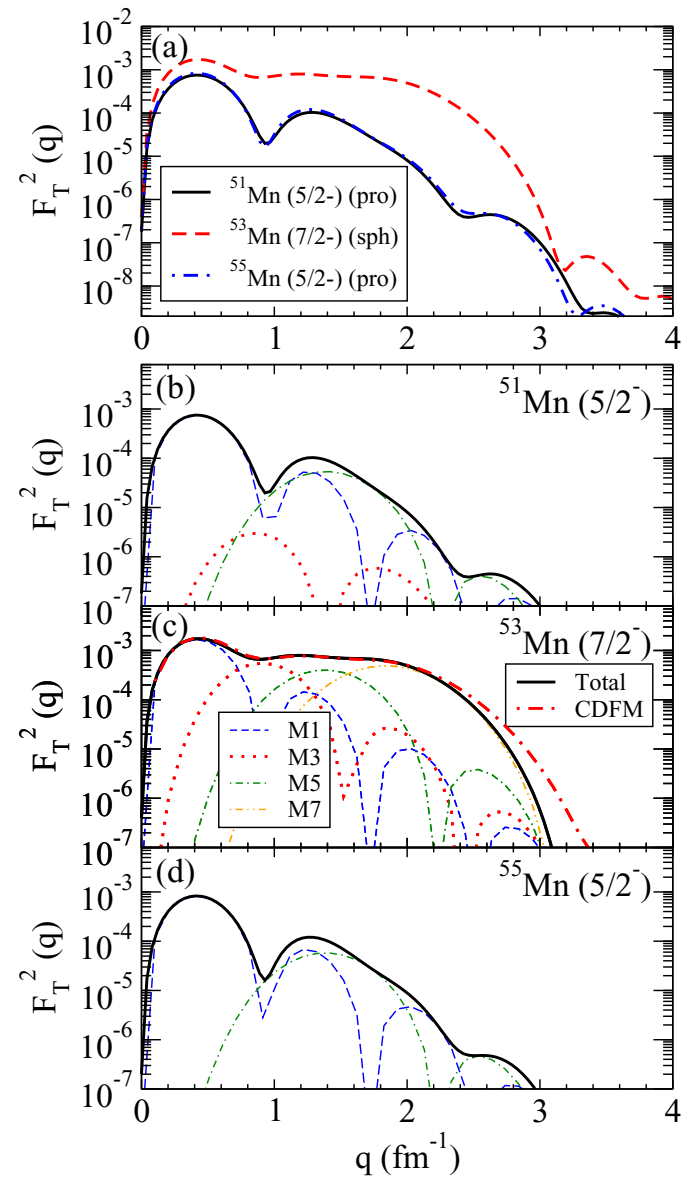

FIG. 4. Same as described in the caption of Fig. 1, but for the $Z=25$ isotopes ${ }^{51,53,55} \mathrm{Mn}$.

\section{Cesium isotopes}

The magnetic form factors of cesium isotopes $(Z=55)$ ${ }^{131,133,135} \mathrm{Cs}$ are plotted in Fig. 6. The experimental angular momentum and spin assignments of the ground states are $5 / 2^{+}$in ${ }^{131} \mathrm{Cs}, 7 / 2^{+}$in ${ }^{133} \mathrm{Cs}$, and $7 / 2^{+}$in ${ }^{135} \mathrm{Cs}$. This change is related to a shape transition from oblate in ${ }^{131} \mathrm{Cs}$ to spherical in ${ }^{133,135} \mathrm{Cs}$, as can be seen in Table I. The spherical shell is now $g_{7 / 2}$ and the oblate Nilsson state is [402]5/2.

A low-lying excited state $7 / 2^{+}$appears at $E=79 \mathrm{keV}$ in ${ }^{131} \mathrm{Cs}$, while $5 / 2^{+}$excited states appear at $E=81 \mathrm{keV}$ and $E=250 \mathrm{keV}$ in ${ }^{133} \mathrm{Cs}$ and ${ }^{135} \mathrm{Cs}$, respectively.

Similarly to the previous case, Fig. 6 shows a clear difference between the magnetic form factors of the spherical and deformed cases. The form factor in ${ }^{131} \mathrm{Cs}$ shows a clear three-peaked structure, while the form factor in the spherical isotopes ${ }^{133,135} \mathrm{Cs}$ is rather smooth because of the enhancement of the intermediate multipoles. As in the case of iodine, the CDFM calculations show little effect on cesium isotopes.

\section{Form factors in isotonic chains}

We consider now several odd- $N$ and even- $Z$ isotonic chains, which are characterized by a change in the spin/parity of their ground states. Namely, we study the following chains: (i) $N=9$ with ${ }^{15} \mathrm{C},{ }^{17} \mathrm{O}$, and ${ }^{19} \mathrm{Ne}$, (ii) $N=11$ with ${ }^{19} \mathrm{O},{ }^{21} \mathrm{Ne}$, 

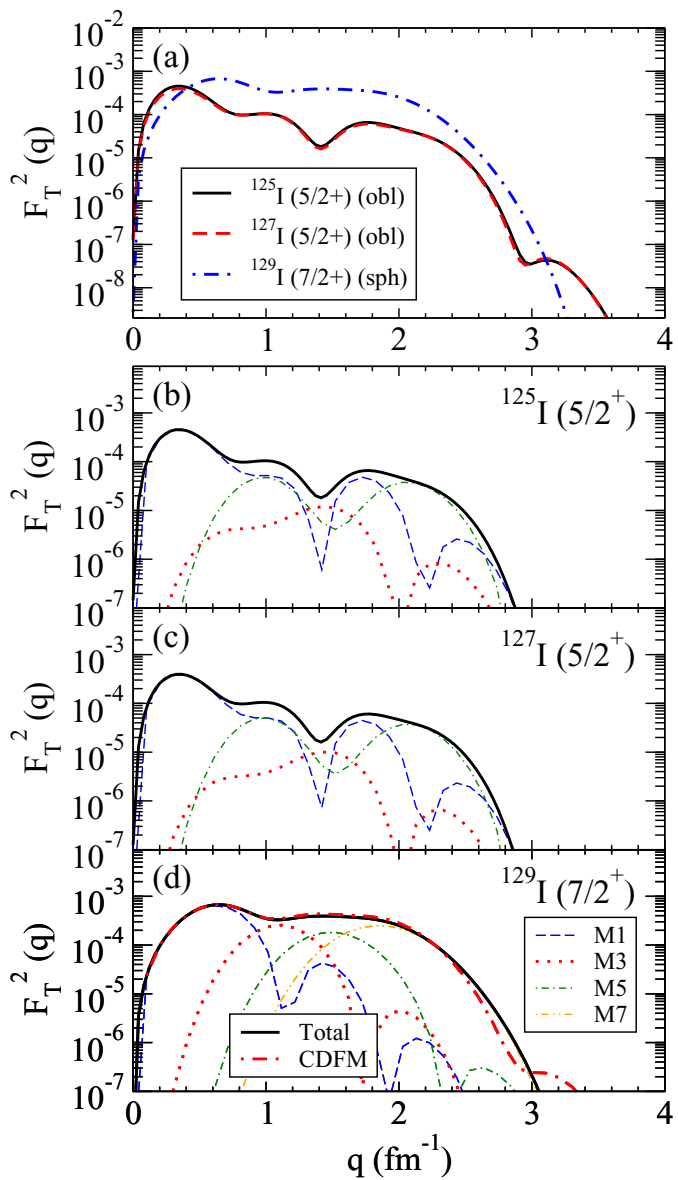

FIG. 5. Same as described in the caption of Fig. 1, but for the $Z=53$ isotopesr ${ }^{125,127,129} \mathrm{I}$.

and ${ }^{23} \mathrm{Mg}$, (iii) $N=25$ with ${ }^{45} \mathrm{Ca},{ }^{47} \mathrm{Ti}$, and ${ }^{49} \mathrm{Cr}$, and (iv) $N=57$ with ${ }^{99} \mathrm{Mo},{ }^{101} \mathrm{Ru}$, and ${ }^{103} \mathrm{Pd}$.

As we shall see, the differences in the magnetic form factors in a given chain are in general larger between deformed isotones with the same $I^{\pi}$ than between deformed isotopes with the same $I^{\pi}$. This is due to the convection current changes induced by the different number of protons in the members of an isotonic chain.

\section{1. $N=9$ isotones}

We show in Fig. 7 the chain of $N=9$ isotones given by ${ }^{15} \mathrm{C},{ }^{17} \mathrm{O}$, and ${ }^{19} \mathrm{Ne}$. The measured spin/parities are $1 / 2^{+}$in ${ }^{15} \mathrm{C}, 5 / 2^{+}$in ${ }^{17} \mathrm{O}$, and $1 / 2^{+}$in ${ }^{19} \mathrm{Ne}$. This change is related to a shape transition from a prolate deformation in ${ }^{15} \mathrm{C}$ to a spherical shape in ${ }^{17} \mathrm{O}$ (related to the $Z=8$ magic number), and to a prolate shape again in ${ }^{19} \mathrm{Ne}$, as can be seen in Table I.

The odd neutron sits naturally in the $d_{5 / 2}$ shell in the spherical nucleus ${ }^{17} \mathrm{O}$, but in the deformed isotones ${ }^{15} \mathrm{C}$ and ${ }^{19} \mathrm{Ne}$ the odd neutron sits in the asymptotic orbital [220]1/2. It is also worth noting that $1 / 2^{+}$excited states are found experimentally in ${ }^{17} \mathrm{O}$ at $E=871 \mathrm{keV}$, and $5 / 2^{+}$excited states are found in ${ }^{15} \mathrm{C}$ and ${ }^{19} \mathrm{Ne}$ at $E=740 \mathrm{keV}$ and $E=238 \mathrm{keV}$, respectively.

Figure 7 shows a large difference between the magnetic form factors of these isotones. Whereas differences between
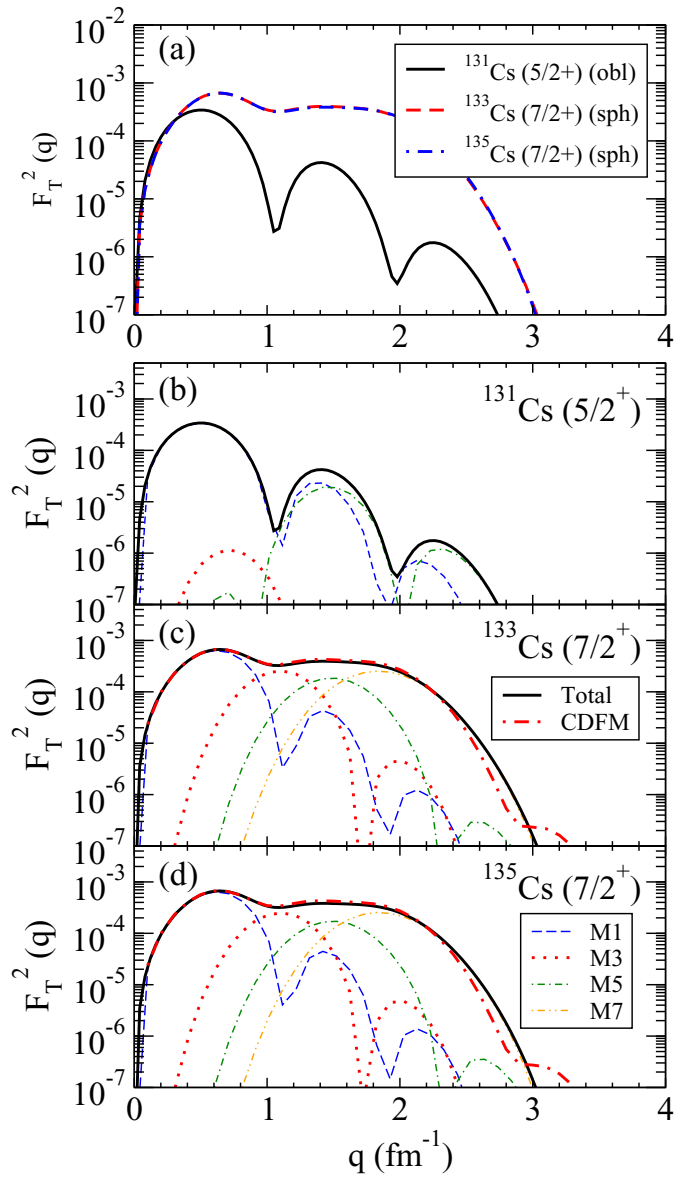

FIG. 6. Same as described in the caption of Fig. 1, but for the $Z=55$ isotopes ${ }^{131,133,135} \mathrm{Cs}$.

the deformed nuclei ${ }^{15} \mathrm{C}$ and ${ }^{19} \mathrm{Ne}$, which are mainly due to the different contributions from the $Z=6$ and $Z=10$ cores, are not very significant, the difference with respect to the spherical nucleus ${ }^{17} \mathrm{O}$ are dramatic and a clear signature of a shape transition. The difference between the form factors for these spherical or deformed isotones is more than one order of magnitude beyond $q=1 \mathrm{fm}^{-1}$.

Our results reproduce very reasonably the measured magnetic form factor in ${ }^{17} \mathrm{O}[15,65]$, especially when correlations calculated within the CDFM are included, that improve the agreement at high momentum transfer. It will be very interesting to check against experiment the predictions for the form factors of those isotones.

\section{2. $N=11$ isotones}

Figure 8 contains the results for the $N=11$ isotones, ${ }^{19} \mathrm{O}$, ${ }^{21} \mathrm{Ne}$, and ${ }^{23} \mathrm{Mg}$. In this chain the ground state of ${ }^{19} \mathrm{O}$ is again spherical $\left(5 / 2^{+}\right)$, related to the $Z=8$ magic number. The odd neutron belongs to the spherical $d_{5 / 2}$ shell. However, both isotones ${ }^{21} \mathrm{Ne}$ and ${ }^{23} \mathrm{Mg}$ are $3 / 2^{+}$prolate states with asymptotic quantum numbers $[211] 3 / 2$. There is a $3 / 2^{+}$excited state in ${ }^{19} \mathrm{O}$ at $E=96 \mathrm{keV}$, and a $5 / 2^{+}$excited state in ${ }^{21} \mathrm{Ne}$ as well as in ${ }^{23} \mathrm{Mg}$ at $E=351 \mathrm{keV}$ and at $E=451 \mathrm{keV}$, respectively.

Similarly to the case of the $N=9$ chain, the comparison of the form factors in Fig. 8 shows that the shape transition 

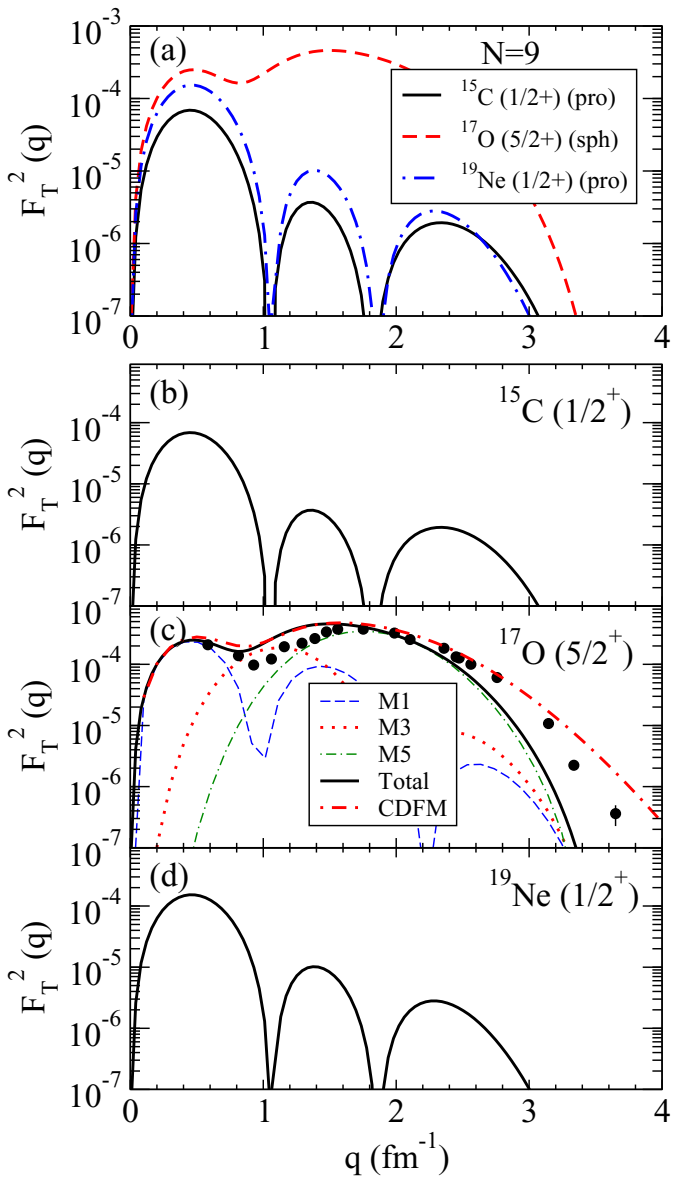

FIG. 7. Same as described in the caption of Fig. 1, but for the $N=9$ isotones ${ }^{15} \mathrm{C},{ }^{17} \mathrm{O}$, and ${ }^{19} \mathrm{Ne}$. Data are taken from Refs. [15,65].

produce quite different form factors that could be easily distinguished experimentally.

\section{3. $N=25$ isotones}

The next example we consider is the isotonic chain $N=$ 25 , including the spherical nucleus ${ }^{45} \mathrm{Ca}(Z=40)$, and the prolate isotones ${ }^{47} \mathrm{Ti}$ and ${ }^{49} \mathrm{Cr} .{ }^{45} \mathrm{Ca}$ has a $7 / 2^{-}$ground state that corresponds to the $f_{7 / 2}$ spherical shell. ${ }^{47} \mathrm{Ti}$ and ${ }^{49} \mathrm{Cr}$ have $5 / 2^{-}$with the odd neutron in the [312]5/2 state.

A $5 / 2^{-}$excited state is found in ${ }^{45} \mathrm{Ca}$ at $E=174 \mathrm{keV}$. Low-lying $7 / 2^{-}$excited state are found in ${ }^{47} \mathrm{Ti}$ and ${ }^{49} \mathrm{Cr}$ at $E=159 \mathrm{keV}$ and $E=272 \mathrm{keV}$, respectively.

Similarly to the previous cases for $N=9$ and $N=11$, the profile of the spherical nucleus in Fig. 9 is very different from the profiles of the deformed isotones. This is a clear signature of a structural change that will be worth exploring experimentally.

\section{4. $N=57$ isotones}

Finally, we study the $N=57$ isotones, ${ }^{99} \mathrm{Mo},{ }^{101} \mathrm{Ru}$, and ${ }^{103} \mathrm{Pd}$, where the shape changes from spherical $1 / 2^{+}$in ${ }^{99} \mathrm{Mo}$ to prolate $5 / 2^{+}$in ${ }^{101} \mathrm{Ru}$ and ${ }^{103} \mathrm{Pd}$, as can be seen in Table I.
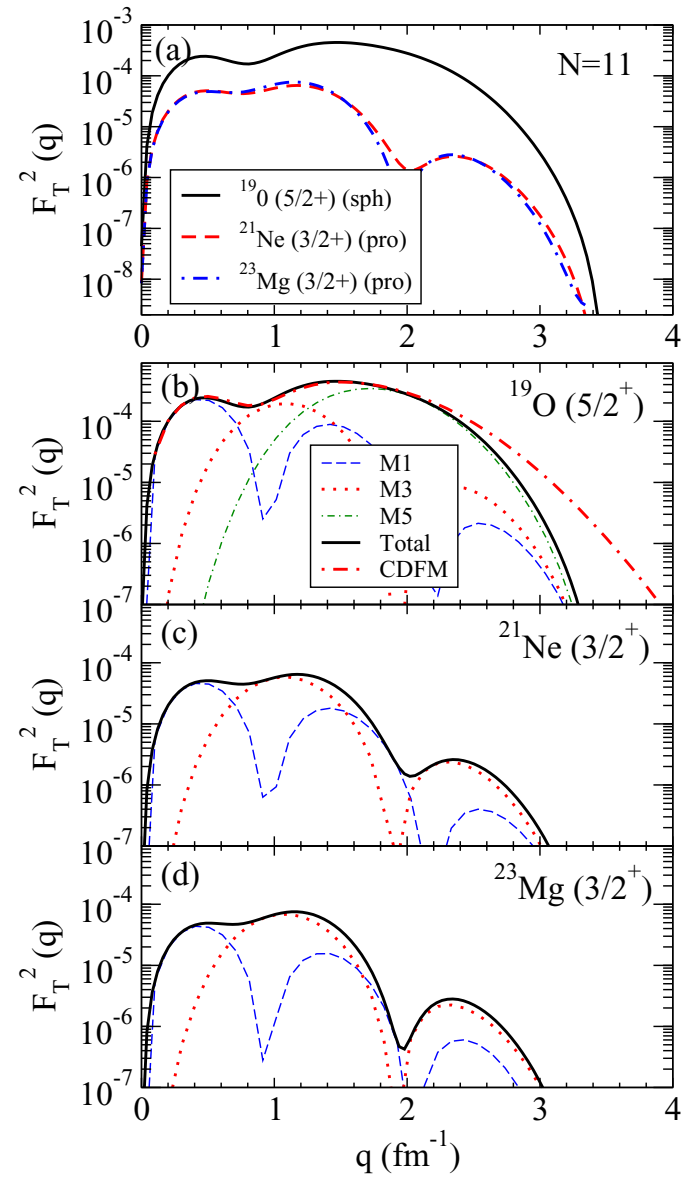

FIG. 8. Same as described in the caption of Fig. 1, but for the $N=11$ isotones ${ }^{19} \mathrm{O},{ }^{21} \mathrm{Ne}$, and ${ }^{23} \mathrm{Mg}$.

Experimentally, a $5 / 2^{+}$excited state is found at $E=98 \mathrm{keV}$ in ${ }^{99} \mathrm{Mo}$ and $1 / 2^{+}$excited states are found at $E=325 \mathrm{keV}$ and $E=504 \mathrm{keV}$ in ${ }^{101} \mathrm{Ru}$ and ${ }^{103} \mathrm{Pd}$, respectively. In the spherical case the odd neutron occupies the $3 s_{1 / 2}$ shell, while in the prolate case the $5 / 2^{+}$states [413]5/2 appear from the degeneracy breaking of $d_{5 / 2}$ and $g_{7 / 2}$ shells.

The form factors in Fig. 10 exhibit significant differences between the spherical and deformed cases, although a threepeaked structure is observed in the three isotones.

\section{CONCLUSIONS}

We have studied the sensitivity of the magnetic form factors, that could be measured in elastic electron scattering, to structural changes of the nucleus. We focus on odd$Z$ and even- $N$ isotopic chains, as well as on even- $Z$ and odd- $N$ isotonic chains, looking for changes in the experimental ground-state spins and parities within a given chain. Nuclear structure calculations based on self-consistent deformed HF + BCS calculations with Skyrme forces have shown that the changes of spin/parity are related to shape transitions.

We have considered first the chains of odd- $A$ vanadium $\left({ }^{49,51,53} \mathrm{~V}\right)$ and aluminum $\left({ }^{25,27,29} \mathrm{Al}\right)$ isotopes as examples of 

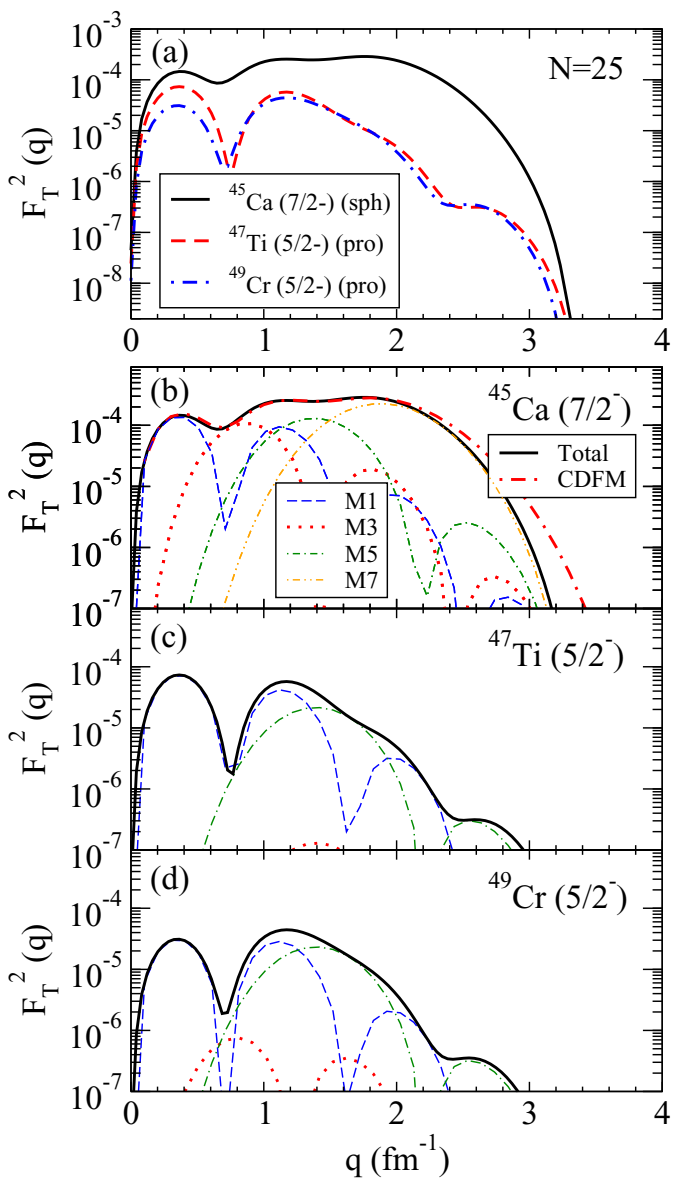

FIG. 9. Same as described in the caption of Fig. 1, but for the $N=25$ isotones ${ }^{45} \mathrm{Ca},{ }^{47} \mathrm{Ti}$, and ${ }^{49} \mathrm{Cr}$.

spherical and deformed nuclei where the ground-state spin and parity is the same in all the members of the chain. This is the expected behavior when the nuclear structure in general and the odd-proton wave function in particular does not change significantly with the addition of an even number of neutrons. We have shown that the magnetic form factors of the isotopes within these chains are basically the same. After these two examples we studied isotopic chains of fluorine $\left({ }^{17,19,21} \mathrm{~F}\right)$, manganese $\left({ }^{51,53,55} \mathrm{Mn}\right)$, iodine $\left({ }^{125,127,129} \mathrm{I}\right)$, and cesium $\left({ }^{131,133,135} \mathrm{Cs}\right)$, as well as isotonic chains with $N=9\left({ }^{15} \mathrm{C},{ }^{17} \mathrm{O},{ }^{19} \mathrm{Ne}\right), N=11\left({ }^{19} \mathrm{O},{ }^{21} \mathrm{Ne},{ }^{23} \mathrm{Mg}\right), N=25$ $\left({ }^{45} \mathrm{Ca},{ }^{47} \mathrm{Ti},{ }^{49} \mathrm{Cr}\right)$, and $N=57\left({ }^{99} \mathrm{Mo},{ }^{101} \mathrm{Ru},{ }^{103} \mathrm{Pd}\right)$. In all of these chains the ground-state spins and parities change within the chain. The corresponding nuclear structure calculations demonstrate the correlation between a change of the ground-state spin and parity and a shape transition within the chain. The results of the calculations of the elastic magnetic form factors exhibit quite different profiles for different deformations that could be interpreted as signatures of shape transitions.

Comparison with experiment in the few stable nuclei where this information is available (see also Ref. [29]) demonstrates the ability of the method to reproduce the data, especially at low momentum transfer and even at higher values beyond

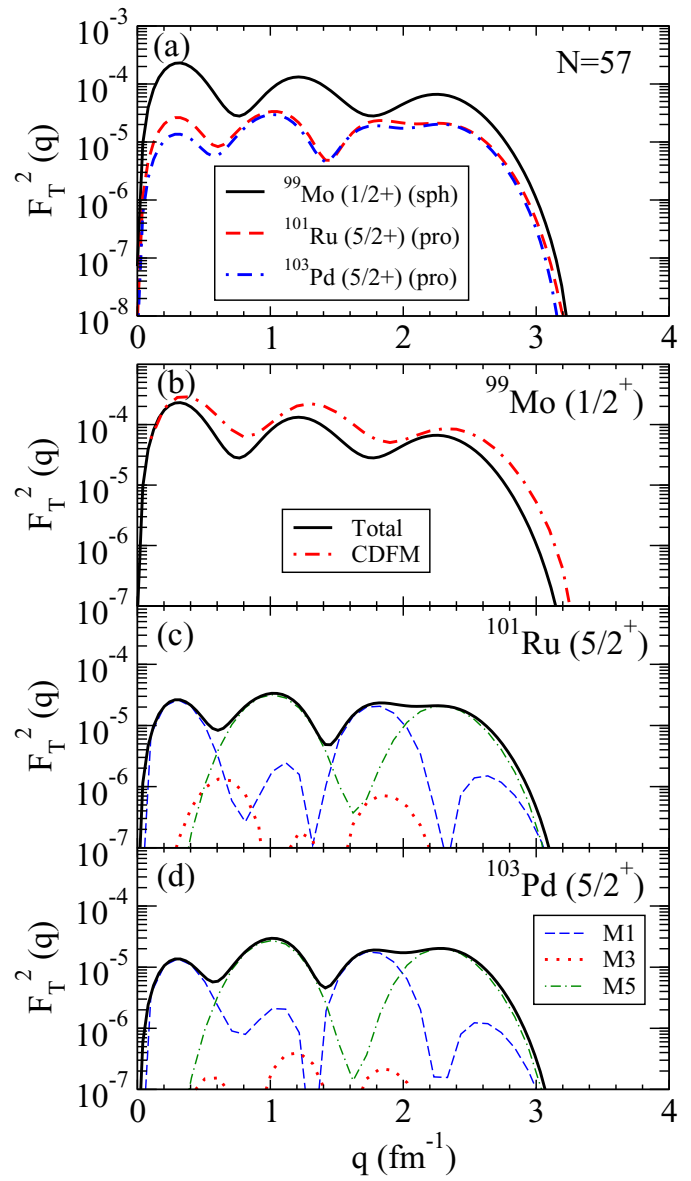

FIG. 10. Same as described in the caption of Fig. 1, but for the $N=57$ isotones ${ }^{99} \mathrm{Mo},{ }^{101} \mathrm{Ru}$, and ${ }^{103} \mathrm{Pd}$.

$2 \mathrm{fm}^{-1}$ when short-range correlations are included. These nucleon-nucleon correlations, taken into account by the CDFM method, shift the tails of the form factors at higher momentum transfer. This comparison is also needed to trust the predictions for unstable nuclei, where no data are available yet.

It would be very interesting to check experimentally the predictions for the magnetic form factors for the isotopic and isotonic chains analyzed here, which have been found to show a shape transition. The present theoretical study is timely, as new experimental electron-scattering facilities are expected to deal soon with unstable nuclei.

\section{ACKNOWLEDGMENTS}

This work was supported by Ministerio de Ciencia e Innovación MCI/AEI/FEDER,UE (Spain) under Contracts No. PGC2018-093636-B-I00 and No. RTI2018-098868-BI00. Two of the authors (D.N.K. and A.N.A.) are grateful for support of the Bulgarian Science Fund under Contract No. KP-06-N38/1. 
[1] R. Hofstadter, Rev. Mod. Phys. 28, 214 (1956).

[2] T. de Forest and J. D. Walecka, Ad. Phys. 15, 1 (1966).

[3] T. W. Donnelly and J. D. Walecka, Ann. Rev. Nucl. Sci. 25, 329 (1975).

[4] J. Heisenberg and H. P. Blok, Ann. Rev. Nucl. Sci. 33, 569 (1983).

[5] H. De Vries, C. W. De Jager, and C. De Vries, At. Data Nucl. Data Tables 36, 495 (1987).

[6] S. Frullani and J. Mougey, Adv. Nucl. Phys. 14, 1 (1984).

[7] J. M. Udias, P. Sarriguren, E. Moya de Guerra, E. Garrido, and J. A. Caballero, Phys. Rev. C 48, 2731 (1993).

[8] K. Heyde, P. von Neumann-Cosel, and A. Richter, Rev. Mod. Phys. 82, 2365 (2010).

[9] P. Sarriguren, E. Moya de Guerra, R. Nojarov, and A. Faessler, J. Phys. G 20, 315 (1994).

[10] T. W. Donnelly, J. Dubach, and I. Sick, Nucl. Phys. A 503, 589 (1989).

[11] O. Moreno, P. Sarriguren, E. Moya de Guerra, J. M. Udias, T. W. Donnelly, and I. Sick, Nucl. Phys. A 828, 306 (2009).

[12] Abrahamyan et al., Phys. Rev. Lett. 108, 112502 (2012).

[13] K. Langanke, G. Martinez-Pinedo, P. von Neumann-Cosel, and A. Richter, Phys. Rev. Lett. 93, 202501 (2004).

[14] A. N. Antonov, M. V. Ivanov, M. K. Gaidarov, E. Moya de Guerra, J. A. Caballero, M. B. Barbaro, J. M. Udias, and P. Sarriguren, Phys. Rev. C 74, 054603 (2006).

[15] T. W. Donnelly and I. Sick, Rev. Mod. Phys. 56, 461 (1984).

[16] E. Moya de Guerra, Phys. Rep. 138, 293 (1986).

[17] E. Moya de Guerra, Ann. Phys. (NY) 128, 286 (1980).

[18] R. A. Radhi, N. T. Khalaf, and A. A. Najim, Nucl. Phys. A 724, 333 (2003).

[19] R. A. Radhi, Eur. Phys. J. A 34, 107 (2007).

[20] K. S. Jassim, A. A. Al-Sammarrae, F. I. Sharrad, and H. A. Kassim, Phys. Rev. C 89, 014304 (2014).

[21] A. A. Al-Sammarraie, F. I. Sharrad, N. Yusof, and H. A. Kassim, Phys. Rev. C 92, 034327 (2015).

[22] Z. Wang, Z. Ren, T. Dong, and X. Guo, Phys. Rev. C 92, 014309 (2015).

[23] E. Moya de Guerra and A. E. L. Dieperink, Phys. Rev. C 18, 1596 (1978).

[24] E. Moya de Guerra and S. Kowalski, Phys. Rev. C 22, 1308 (1980).

[25] E. Graca, P. Sarriguren, D. Berdichevsky, D. W. L. Sprung, E. Moya de Guerra, and M. Nishimura, Nucl. Phys. A 483, 77 (1988).

[26] P. Sarriguren, E. Graca, D. W. L. Sprung, E. Moya de Guerra, and D. Berdichevsky, Phys. Rev. C 40, 1414 (1989).

[27] D. Berdichevsky, P. Sarriguren, E. Moya de Guerra, M. Nishimura, and D. W. L. Sprung, Phys. Rev. C 38, 338 (1988).

[28] E. Moya de Guerra, P. Sarriguren, J. A. Caballero, M. Casas, and D. W. L. Sprung, Nucl. Phys. A 529, 68 (1991).

[29] P. Sarriguren, D. Merino, O. Moreno, E. Moya de Guerra, D. N. Kadrev, A. N. Antonov, and M. K. Gaidarov, Phys. Rev. C 99, 034325 (2019).

[30] J. M. Yao, M. Bender, and P.-H. Heenen, Phys. Rev. C 91, 024301 (2015).

[31] L. Wang, J. Liu, T. Liang, Z. Ren, C. Xu, and S. Wang, J. Phys. G 47, 025105 (2020).

[32] K. Heyde and J. L. Wood, Rev. Mod. Phys. 83, 1467 (2011).

[33] R. Rodriguez-Guzman, P. Sarriguren, and L. M. Robledo, Phys. Rev. C 83, 044307 (2011).
[34] T. Suda and H. Simon, Prog. Part. Nucl. Phys. 96, 1 (2017).

[35] https://fair-center.eu/for-users/experiments/nustar/experiments/ elise.html.

[36] http://www.riken.jp/en/research/labs/rnc/instrum_dev/scrit/.

[37] A. N. Antonov et al., Nucl. Inst. and Meth. A 637, 60 (2011).

[38] E. Enokizono, T. Onishi, and K. Tsukada, Nuclear Physics News 28, 18 (2018).

[39] T. Ohnishi et al., Proc. Sci. INPC2016, 088 (2017).

[40] K. Tsukada, A. Enokizono, T. Ohnishi, K. Adachi, T. Fujita, M. Hara, M. Hori, T. Hori, S. Ichikawa, K. Kurita, K. Matsuda, T. Suda, T. Tamae, M. Togasaki, M. Wakasugi, M. Watanabe, and K. Yamada, Phys. Rev. Lett. 118, 262501 (2017).

[41] E. Garrido and E. Moya de Guerra, Nucl. Phys. A 650, 387 (1999); Phys. Lett. B 488, 68 (2000).

[42] A. N. Antonov, D. N. Kadrev, M. K. Gaidarov, E. Moya de Guerra, P. Sarriguren, J. M. Udias, V. K. Lukyanov, E. V. Zemlyanaya, and G. Z. Krumova, Phys. Rev. C 72, 044307 (2005).

[43] T. Dong, Z. Ren, and Y. Guo, Phys. Rev. C 76, 054602 (2007); 78, 044332 (2008).

[44] X. Roca-Maza, M. Centelles, F. Salvat, and X. Viñas, Phys. Rev. C 87, 014304 (2013).

[45] R. A. Radhi, A. A. Alzubadi, and A. H. Ali, Phys. Rev. C 97, 064312 (2018).

[46] T. Liang, J. Liu, Z. Ren, C. Xu, and S. Wang, Phys. Rev. C 98, 044310 (2018).

[47] G. G. Simon, Ch. Schmitt, F. Borkowski, and V. H. Walther, Nucl. Phys. A 333, 381 (1980).

[48] H. Chandra and G. Sauer, Phys. Rev. C 13, 245 (1976).

[49] D. R. Yennie, D. G. Ravenhall, and R. N. Wilson, Phys. Rev. 95, 500 (1954).

[50] D. Vautherin and D. M. Brink, Phys. Rev. C 5, 626 (1972); D. Vautherin, ibid. 7, 296 (1973).

[51] E. Chabanat, P. Bonche, P. Haensel, J. Meyer, and R. Schaeffer, Nucl. Phys. A 635, 231 (1998).

[52] A. N. Antonov, P. E. Hodgson, and I. Zh. Petkov, Nucleon Momentum and Density Distributions in Nuclei (Clarendon Press, Oxford, 1988); Nucleon Correlations in Nuclei (SpringerVerlag, Berlin, 1993).

[53] D. N. Kadrev, A. N. Antonov, M. V. Stoitsov, and S. S. Dimitrova, Int. J. Mod. Phys. E 5, 717 (1996).

[54] P.-O. Löwdin, Phys. Rev. 97, 1474 (1955).

[55] A. N. Antonov, V. A. Nikolaev, and I. Zh. Petkov, Bulg. J. Phys. 6, 151 (1979); Z. Phys. A 297, 257 (1980); 304, 239 (1982).

[56] A. N. Antonov, D. N. Kadrev, and P. E. Hodgson, Phys. Rev. C 50, 164 (1994).

[57] I. Angeli and K. P. Marinova, At. Data Nucl. Data Tables 99, 69 (2013).

[58] N. J. Stone, At. Data Nucl. Data Tables 90, 75 (2005).

[59] K. Arita, A. Enomoto, S. Oguro, Y. Mizuno, T. Nakazato, S. Ohsawa, T. Saito, T. Terasawa, and Y. Torizuka, Phys. Rev. C 23, 1482 (1981).

[60] S. Platchkov et al., Phys. Lett. B 131, 301 (1983).

[61] L. Lapikas, A. E. L Dieperink, and G. Box, Nucl. Phys. A 203, 609 (1973).

[62] G. C. Li et al., Phys. Lett. B 32, 317 (1970).

[63] C. F. Williamson, F. N. Rad, S. Kowalski, J. Heisenberg, Hall Crannell, J. T. O'Brien, and H. C. Lee, Phys. Rev. Lett. 40, 1702 (1978).

[64] A. J. H. Donné et al., Nucl. Phys. A 455, 453 (1986).

[65] M. V. Hynes et al., Phys. Rev. Lett. 42, 1444 (1979). 